\title{
Regarding the Remote-Knot Technique
}

\author{
Thomas C. Wiener
}

Published online: 12 September 2009

(c) Springer Science+Business Media, LLC and International Society of Aesthetic Plastic Surgery 2009

I would like to respond to Drs. Maia and Azevedo letter [1] about in my article [2]. Overall, I would agree with their analysis that the remote-knot technique may not hold any advantage in many types of plastic surgery incision closures. In the development of this technique, I too originally used the technique described by Dr. Azevedo. The main reason for going beyond this and removing the knot from the length of the surgical incision was based on observations in breast augmentation.

If there is a knot extrusion where there is an underlying device such as a breast implant, and in particular with the often thin tissue coverage at the inframammary location, then the problem of either contamination of the knot, with subsequent tracking of bacteria and infection into the breast pocket, or outright wound breakdown would be disasterous. In removing the suture knot and placing it in a remote location, even if only $5 \mathrm{~mm}$ away, provides greater soft tissue coverage in the event of a knot extrusion and may help prevent this problem because the surrounding tissue is thicker and more robust. Remote-knot closure, when properly performed, causes the suture to travel from the knot through unbroken soft tissue before entering the confines of the surgical incision.

There has been only one knot extrusion case since developing this technique and this was treated by keeping the knot covered with topical antibiotic ointment and a bandage until it healed. This would be done for extrusions within the first 3 weeks of surgery; beyond that, I would clip the exposed part of the knot and keep it covered with antibiotic ointment until it heals. I and the patients have not found the stab incision to be objectionable and have yet to have any patient inquire about it, as it heals quite well.

Any surgery that has thin tissue coverage with underlying vital components-either tissue components or implanted devices-may benefit from the remote-knot technique. In having performed over 4000 breast augmentations, I have found the remote-knot technique to be the most reliable incision closure I have used.

\section{References}

1. Maia M, Azevedo L (2009) Intradermal double-layer closure with suture knot contained within incision-easy, fast, and safe. Aesthetic Plast Surg 33. doi:10.1007/s00266-009-9403-3

2. Wiener TC (2009) A modified incision closure for breast augmentation: remote-knot technique. Aesthetic Plast Surg $33: 110-112$

T. C. Wiener ( $\square)$

2323 Clear Lake City Blvd., \#152, Houston, TX 77062, USA

e-mail: thomwien@aol.com 